\title{
Could the Topping-Off Technique Be the Preventive Strategy against Adjacent Segment Disease after Pedicle Screw-Based Fusion in Lumbar Degenerative Diseases? A Systematic Review
}

\author{
Po-Hsin Chou, ${ }^{1,2,3}$ Hsi-Hsien Lin, ${ }^{1,2}$ Howard S. An, ${ }^{3}$ Kang-Ying Liu, ${ }^{1,2}$ \\ Wei-Ren Su, ${ }^{4}$ and Cheng-Li Lin ${ }^{3,4}$ \\ ${ }^{1}$ Department of Orthopedics and Traumatology, Taipei Veterans General Hospital, Taipei, Taiwan \\ ${ }^{2}$ School of Medicine, National Yang-Ming University, Taipei, Taiwan \\ ${ }^{3}$ Department of Orthopaedic Surgery, Rush University Medical Center, Chicago, IL, USA \\ ${ }^{4}$ Department of Orthopaedic Surgery, National Cheng Kung University Hospital, College of Medicine, \\ National Cheung Kung University, Tainan, Taiwan
}

Correspondence should be addressed to Cheng-Li Lin; jengli94@gmail.com

Received 25 July 2016; Accepted 10 November 2016; Published 22 February 2017

Academic Editor: Jiancheng Zeng

Copyright (C) 2017 Po-Hsin Chou et al. This is an open access article distributed under the Creative Commons Attribution License, which permits unrestricted use, distribution, and reproduction in any medium, provided the original work is properly cited.

\begin{abstract}
The "topping-off" technique is a new concept applying dynamic or less rigid fixation such as hybrid stabilization device (HSD) or interspinous process device (IPD) for the purpose of avoiding adjacent segment disease (ASD) proximal to the fusion construct. A systematic review of the literature was performed on the effect of topping-off techniques to prevent or decrease the occurrence of ASD after lumbar fusion surgery. We searched through major online databases, PubMed and MEDLINE, using key words related to "topping-off" technique. We reviewed the surgical results of "topping-off" techniques with either HSD or IPD, including the incidence of ASD at two proximal adjacent levels (index and supra-adjacent level) as compared to the fusion alone group. The results showed that the fusion alone group had statistically higher incidence of radiographic (52.6\%) and symptomatic (11.6\%) ASD at the index level as well as higher incidence (8.1\%) of revision surgery. Besides, the HSD (10.5\%) and fusion groups (24.7\%) had statistically higher incidences of radiographic ASD at supra-adjacent level than the IPD (1\%). The findings suggest that the "topping-off" technique may potentially decrease the occurrence of ASD at the proximal motion segments. However, higher quality prospective randomized trials are required prior to wide clinical application.
\end{abstract}

\section{Introduction}

Fusion surgery has been shown to improve functional outcomes in appropriately selected symptomatic patients with various degenerative lumbar disorders $[1,2]$. However, adjacent segment disease (ASD) is still a significant problem following rigid spinal fixation [3, 4]. Fusion surgery aims to relieve symptom from degenerative or unstable motion segments. There is increase in range of motion and stress at the upper adjacent level after rigid fixation $[3,5]$, which is one of many factors, contributing to the development of ASD.

The incidence of radiographic ASD ranges from $5.2 \%$ to $100 \%$, depending on patient population, follow-up duration, the imaging used for evaluation, and definition of ASD [4].
The symptomatic ASD ranged from 5.2\% to $18.5 \%$ as reported by Park et al. [4]. Ghiselli et al. [3] reported the rate of symptomatic ASD following either decompression or fusion was predicted to be $16.5 \%$ at 5 years and $36.1 \%$ at 10 years.

Generally, symptomatic ASD in patients who failed in conservative treatment needs revision surgery to relieve symptoms. However, some studies reported relatively modest results in patients who received revision surgery for symptomatic ASD [6, 7]. Regarding the location of ASD, Aota et al. [8] demonstrated that ASD occurred in $24.6 \%$ of the cases proximal to lumbar fusion and $2.6 \%$ of the cases distal to fusion and a similar trend, reported by Etebar and Cahill [9]. It is important for surgeons to carefully evaluate the proximal adjacent disc above fusion levels before surgery in order to 
lower the occurrence of ASD. The "topping-off" technique with either hybrid stabilization device (HSD) or interspinous process devices (IPD) might be one of the solutions.

This "topping-off" technique refers to application of hybrid dynamic pedicle screw construct or interspinous process device above the fused segments. This technique provides a transitional zone between caudal rigid fused construct and cephalad mobile/unfused segments, which may decrease the incidence of ASD [10,11]. The rationale of this technique is that the semirigid zone provides a gradual transition from the rigid to mobile segments to lessen stress concentration at the adjacent level. Khoueir et al. [12] classified posterior dynamic stabilization devices into three categories: (1) hybrid stabilization device with pedicle screw/rod construct such as $\mathrm{DTO}^{\circledR}$ and Dynesys (we defined it as HSD in this manuscript); (2) interspinous process devices (IPD) such as Wallis, XSTOP, DIAM, and Coflex; (3) total facet replacement system. Because of the lack of evidence in the literature on total facet implants, we focused on the former two devices of HSD and IPD in our literature reviews.

To our knowledge, systematic review investigating the "topping-off" technique with HSD or IPD to prevent ASD following lumbar fusion surgery has not been done. This manuscript reviews the surgical results of "topping-off" techniques and compares the incidence of ASD at proximal two adjacent levels among HSD, IDP, and fusion alone group.

\section{Materials and Methods}

We followed the methodological guidelines outlined by the Transparent Reporting of Systematic Reviews and MetaAnalyses (PRISMA) [19, 20] to conduct this systematic review.

2.1. Literature Search and Selection. A literature review of clinical studies published from January 2007 to December 2015 was conducted. The articles written in English were included. We completed a search into National Center for Biotechnology Information databases using PubMed/ MEDLINE, with keywords and Boolean operators. The search strategy for publications was of "Topping-off", "hybrid stabilization", "hybrid stabilization device", "hybrid stabilisation", "hybrid fixation", and "interspinous process device" AND "fusion", "lumbar spine", "adjacent segment disease", and "adjacent segment degeneration". Editorials and commentaries from major neurosurgical and orthopaedic journals were also reviewed to gather further information on this topic. Furthermore, we searched and reviewed the relevant articles on the reference list for further information. We only included studies published in SCI (scientific citation index) journals.

2.2. Methodological Quality Assessment. Full-text versions of all included articles were downloaded and assessed for potential bias by two independent reviewers (PC \& CL). The National Heart Lung and Blood Institute (NIH) quality assessment tool for case series studies [21] was used to assess the methodological quality of the selected studies. This categorises studies as either good, fair, or poor. Encountering any disagreement, we made a consensus by discussion within the review team.

2.3. Article Selection and Data Extraction. We collected clinical trials studying the effect of hybrid stabilization or proximal IPD implantation to prevent ASD after lumbosacral fusion surgery. Many clinical studies were initially selected including prospective, retrospective studies or case series with or without comparison group (fusion alone). The problems adjacent to fusion levels or ASD were considered as primary outcomes. After reviewing the titles and abstracts of collected studies, we then determined if the content of the studies was suitable for retrieval. The studies in which the average patient follow-up time was less than 24 months or the number of patients was less than 20 were not considered.

Two authors independently extracted data from the articles. We contacted the authors of the studies for the uncertain details. The following data were extracted: (1) participant demographics; (2) indication for surgeries; (3) adjacent segment degeneration; (4) radiographical and clinical outcomes; (5) implant-related complications and other outcomes. Details of ASD following fusion surgery and required revision surgery were further analyzed among the three groups. Only ASD that were specifically stated as having occurred or not having occurred in the articles were used in the analysis. ASD were not assumed to be absent just because they were not discussed (Table 4).

2.4. Statistical Analysis. For statistical analysis, quantitative data are described by the mean, range, and standard deviation if available; qualitative data are described as counts and percentages. We used chi-square test with the Yates continuity correction to evaluate the incidence or proportion in the comparative groups in the parameters. A $p$ value of $<0.05$ indicated statistical significance. All statistical computation has been performed with the SPSS for Windows statistical package (version 21.0, Chicago, Illinois).

\section{Results}

3.1. Identified Trials. A flow chart describing the procedure of study selection is shown in Figure 1. The search yielded 393 articles of prospective or retrospective case series. No additional studies were found manually. All studies had abstracts screened and assessed for eligibility. Thirteen fulltext articles were retrieved and appraised for eligibility. Eventually 366 patients from 6 articles, 2 prospective [12, $14]$ and 4 retrospective [13, 15-17], were included in our systematic review. The methodological quality as measured by the NIH quality assessment tool was high with all studies assessed as good. The level of evidence for these selected articles was also analyzed (Table 1).

3.2. Study Characteristics and Outcomes. The relevant characteristics for each included study are summarized in Table 1. Regarding the level of evidence, there were two papers of level II [14, 18], three papers of level III $[15,16,22]$, and one paper of level IV [13]. Every particular indication 


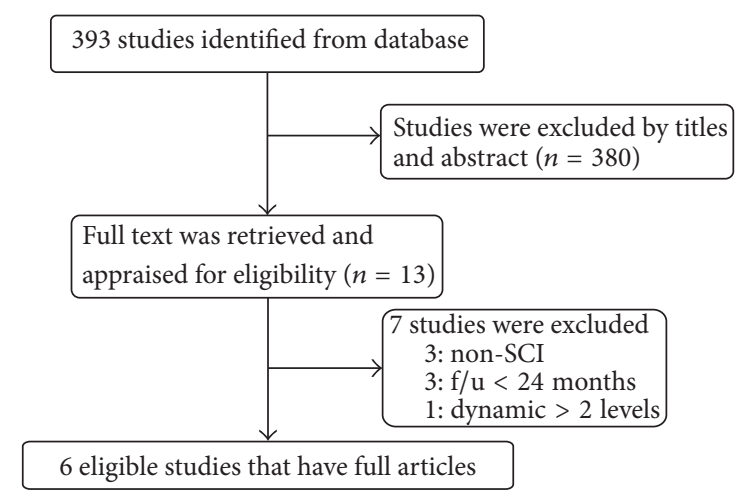

FIGURE 1: The flow chart for manuscript selection.

for "topping-off" surgery was reported in all studies. Some degree of adjacent disc degeneration was the main reason for dynamic stabilization above fusion construct in most $(5 / 6)$ studies. Location for topping-off stabilization was illustrated in 5 studies, located from L1/2 to L4/5. The methodology for evaluating radiographic and clinical results was not consistent in all studies. The evaluation tools and results in each study are summarized in Tables 2 and 3, respectively, for radiographic parameters and functional outcomes. The radiographic evaluation tools used in these studies are disc height, foraminal height, and UCLA grade obtained from plain radiography and Pfirrmann's classification and Modic grade obtained from MRI images. It is difficult to compare the radiographic results among these studies because of the inconsistency of evaluation tools (Table 2). The clinical outcome was evaluated with visual analogue score (VAS) for back or leg, Oswestry Disability Index (ODI), and short form (36) health survey (SF-36). Ultimately, all studies revealed that the clinical outcomes improved significantly postoperatively (Table 3).

3.3. Adjacent Segment Disease. The demographic data and results of ASD for the topping-off techniques and fusion alone group were listed in Table 4. There were 95 patients in HSD group, 98 patients in IPD group, and 173 patients in fusion alone group with a mean age of 62.7, 64.9, and 60.5, respectively. The number of fused vertebrae was 2 in HSD group, 3.4 in IPD group, and 2.5 in fusion alone group. The mean follow-up time was $42.8,47.2$, and 50.4 months in each group. The details of adjacent segment disease for topping-off techniques and fusion alone group are shown in Tables 5 and 6 , respectively. The definitions of "index level" and "supraadjacent level" were illustrated in Figure 2.

3.3.1. ASD at the Index Level. The index level was defined as the level of HSD or IPD or the adjacent level above fusion. The difference in the incidence of radiographic or symptomatic ASD at the index level was statistically significant among the three groups. The fusion group presented statistically higher percentage of symptomatic ASD (11.6\% or probably higher as some papers defined ASD requiring revision surgery for symptomatic ASD) and radiographic ASD (52.6\%) as well as

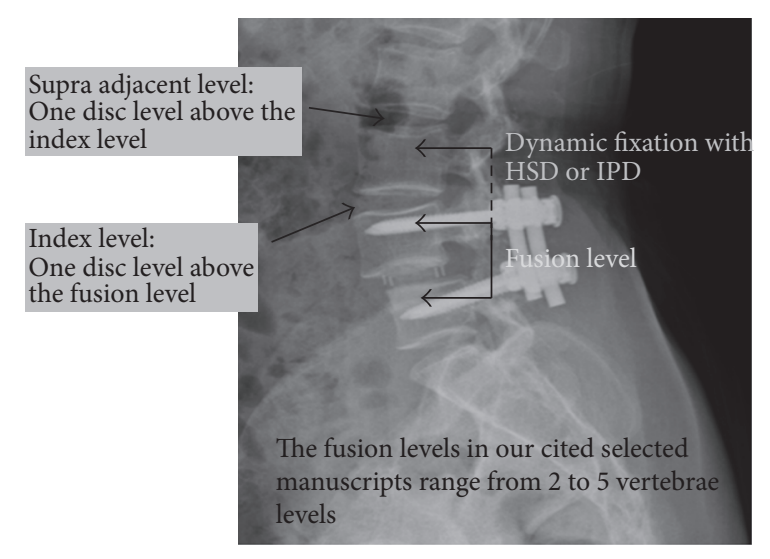

Figure 2: The definitions of "index level" and "supra-adjacent level" in our manuscript. "Index level" represents the disc level just above the fusion construct. "Supra-adjacent level" represents one disc level above the index level. The fusion levels in selected manuscripts ranged from 2 to 5 vertebrae levels, which were not, respectively, presented herein.

revision surgery for ASD (8.1\%) as compared to "topping-off" groups ( $p=0.003, p<0.001$, and $p=0.008$ resp.).

3.3.2. ASD at Supra-Adjacent Level. The supra-adjacent level was defined as the level above index level. Interestingly, the HSD (10.5\%, 7 out of 95 patients) and fusion groups $(24.7 \%$, 20 out of 81 patients) had higher incidences of radiographic ASD at supra-adjacent level than in the IPD (1\%, 1 out of 98 patients) $(p<0.001)$. The fusion alone group still had a higher incidence of ASD at supra-adjacent level as compared to $\operatorname{HSD}(p<0.05)$.

3.4. Implants-Related Complications in HSD or IPD. No implant-related complication was reported in all IPD group. Regarding the HSD group, a patient needed revision surgery after 26 months because of a clinically symptomatic dislocation of the Dynesys screws. This patient was excluded from further analysis because dynamic stabilization was removed during revision surgery.

\section{Discussion}

Accelerated degeneration at adjacent segments above or below lumbar spinal fusion site has been a significant problem in clinical practice. In this review, we focused on the cephalad "topping-off" techniques either HSD or IPD and compared with the fusion alone groups, as these newer techniques are controversial. Our review revealed the potential of these "topping-off" techniques in decreasing the incidence of ASD after fusion surgery.

4.1. The Mechanism of ASD. While rigid fixation improves the fusion rate and functional outcomes $[1,2]$, many studies have reported the increased prevalence of adjacent motion segment degeneration following lumbar fusion $[3,4,7]$. 


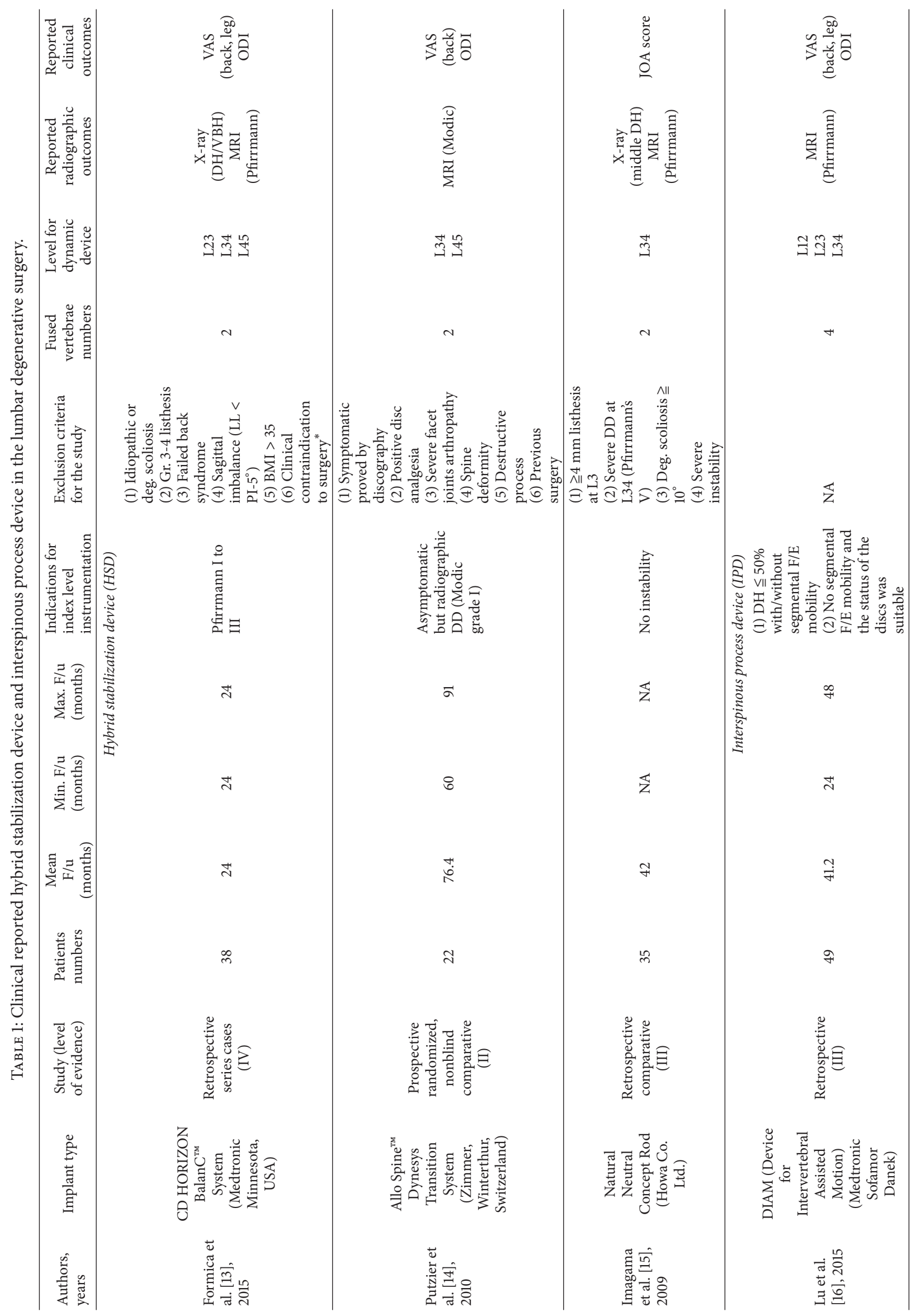




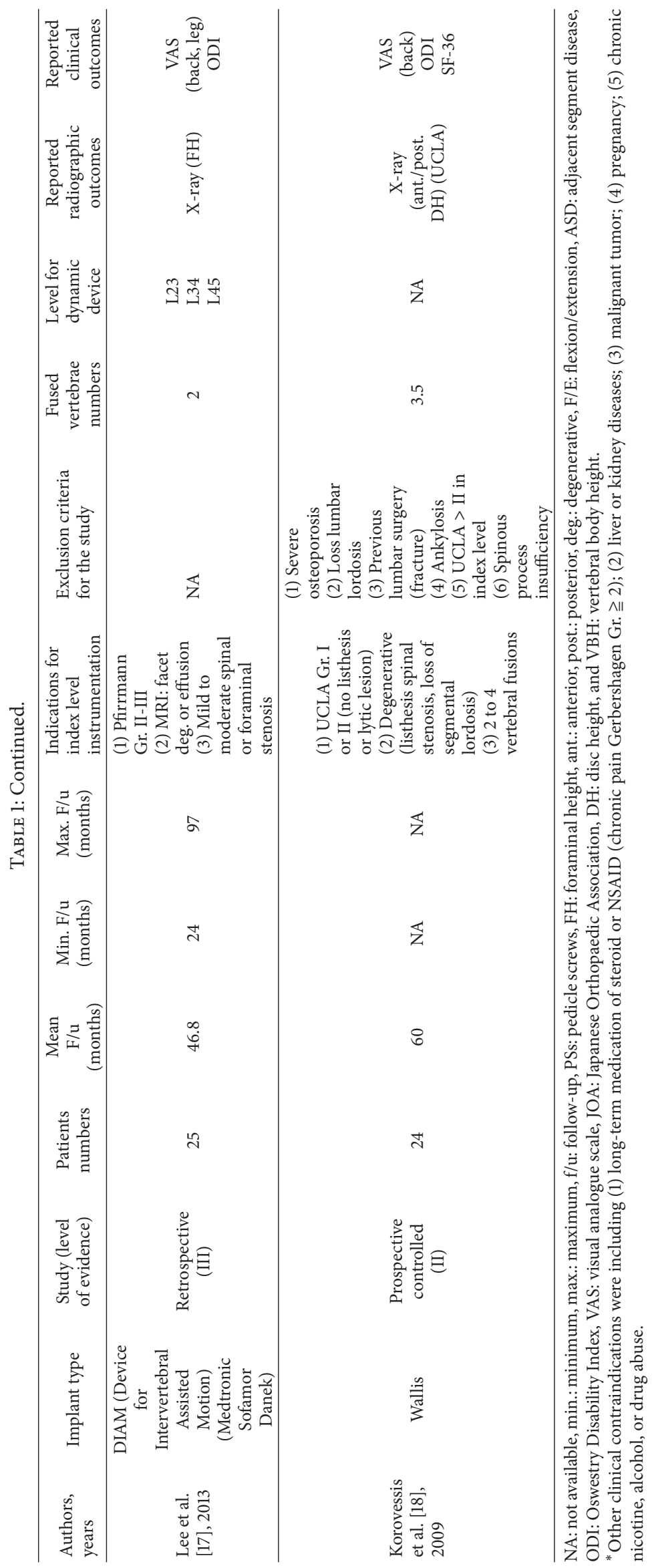




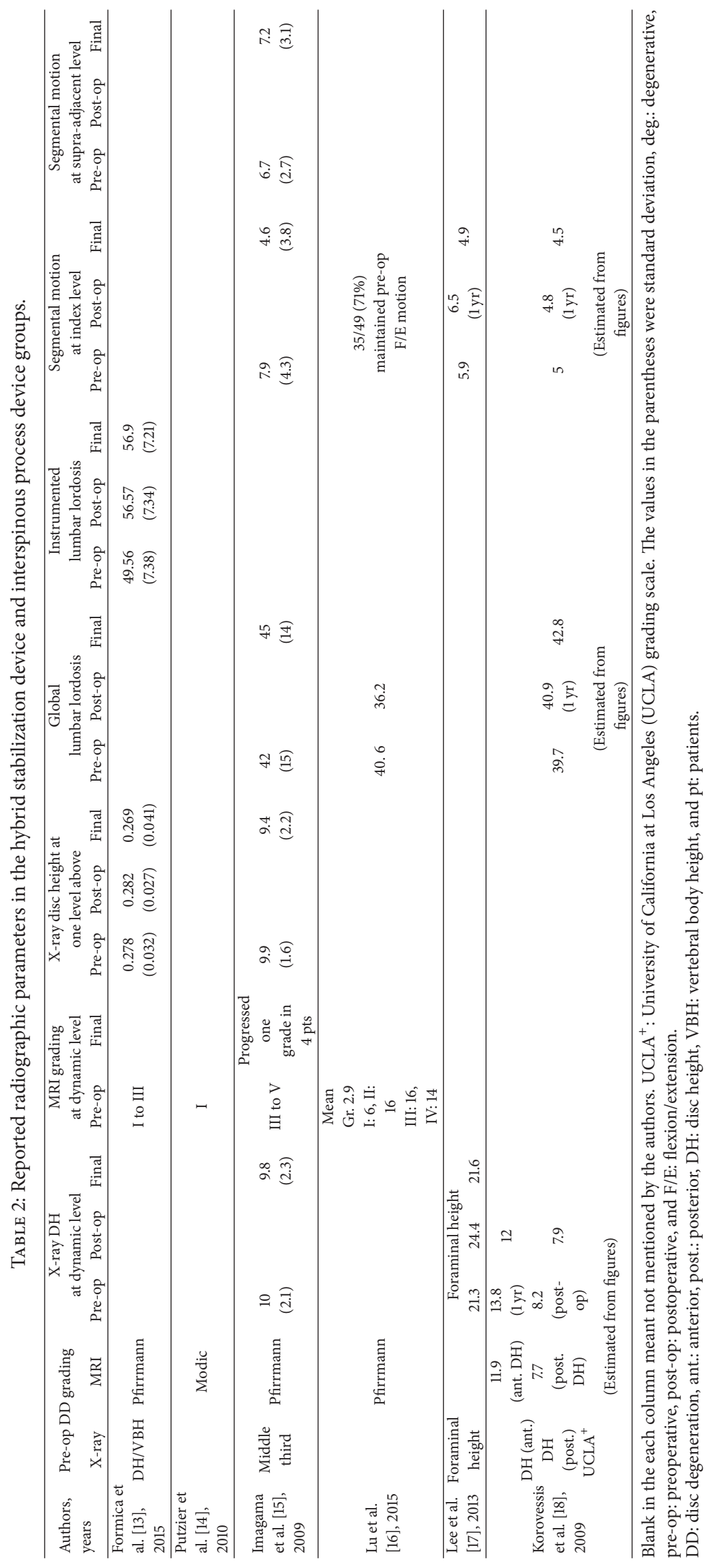




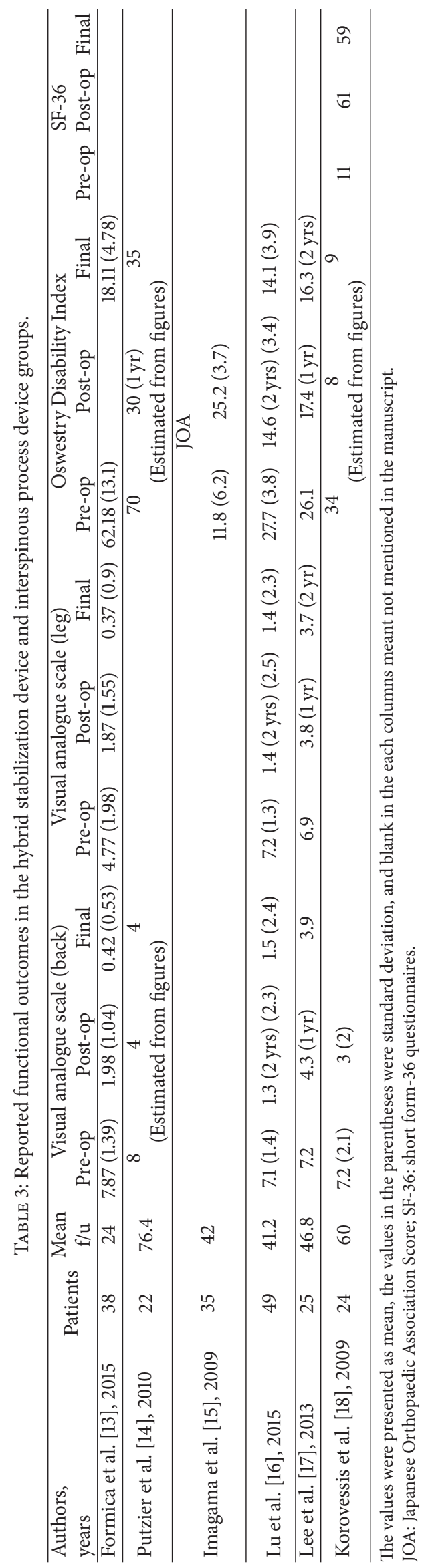


TABLE 4: Data in the hybrid stabilization device, interspinous process device, and fusion groups.

\begin{tabular}{|c|c|c|c|c|}
\hline & Hybrid stabilization device & Interspinous process device & Fusion & $p$ value \\
\hline Numbers of patients & 95 & 98 & 173 & NA \\
\hline $\operatorname{Age}(y / o)^{+}$ & 62.7 & 64.9 & 60.5 & NA \\
\hline Male/female & NA & NA & NA & NA \\
\hline Numbers of fused vertebrae & 2 & 3.4 & 2.45 & NA \\
\hline Follow-up (months) & 42.8 & 47.2 & 50.4 & NA \\
\hline \multicolumn{5}{|l|}{ Adjacent segment disease (ASD) } \\
\hline Symptomatic ASD at index level & 0 & $5(5.1 \%)$ & $20^{++}(11.6 \%)$ & 0.003 \\
\hline Radiographic ASD at index level & $12(12.6 \%)$ & $10(10.2 \%)$ & $91(52.6 \%)$ & $<0.001$ \\
\hline Symptomatic ASD at supra-adjacent level & 0 & 0 & 0 & - \\
\hline Radiographic ASD at supra-adjacent level & $7(10.5 \%)$ & $1(1 \%)$ & $20 / 81^{*}(24.7 \%)$ & $<0.001$ \\
\hline Revision surgery for ASD & 0 & $3(3 \%)$ & $14(8.1 \%)$ & 0.008 \\
\hline
\end{tabular}

The bold numbers in the $p$ values indicated statistical significance. NA indicates not available.

${ }^{+}$The authors did not exclude those who lost follow-up in the demographic results. The mean age was just estimated.

${ }^{++}$Two papers only mentioned numbers of revision surgeries for symptomatic ASD but did not mention numbers of symptomatic ASD. (The result might be underestimated.)

* Only 3 cited manuscripts reported their results.

Although clinical studies investigated risks factors predisposed in the progression of ASD [5, 8, 9, 23-30], the exact pathogenesis of ASD remains uncertain. Biomechanical and clinical studies have suggested the compensatory loading transfer [31] and increased range of motion [3,5] at upper adjacent level after rigid fixation. Regarding the intradiscal pressure (IDP) at proximal adjacent disc (PAD) following rigid fixation, Cunningham et al. [32] reported an increase of IDP up to $45 \%$ on axial compression and anterior flexion loading motion in comparison to normal disc. Weinhoffer et al. [33] also reported a significant increase of IDP at PAD following instrumentation in a simulated fusion model. The authors mentioned increased IDP may alter the metabolic status and further play an important role in the pathogenesis of ASD. However, there are several clinical studies suggesting that ASD is part of a normal degenerative process rather the altered biomechanical stress on the adjacent disc $[34,35]$.

4.2. The Risk Factors for ASD. There are many papers on the risks factors for ASD. These risk factors include patient's age $[8,9,24]$, postmenopausal status [9], sagittal mal-alignment $[5,25,26]$, multiple level fusion [23, 28, 29], posterior interbody fusion [24], iatrogenic injury to the facets of the adjacent segment $[8,30]$, and preexisting disc degeneration [36]. There are other papers in the literature supporting or contradicting these risk factors [4]. Kumar et al. [37] reported that gender, different types of fusion (posterior fusion versus combined posterolateral and posterior interbody fusion), and fusion level (fusions extending down to the sacrum versus fusions stopped at short of the sacrum) are not risk factors for ASD. In addition, Rahm and Hall [24] reported a negative correlation between sagittal alignment and incidence of ASD. The inconsistent conclusions are as a result of retrospective selection bias, limited follow-up time, or different methodology evaluating ASD. The progression of ASD following lumbar spine fusion is obviously multifactorial, and further research can help identify and quantify the contributing risk factors for ASD.
4.3. Intervals from Fusion Surgery to Revision Surgery for Symptomatic ASD. Based on Lee et al. [22], Kumar et al. [5], and Aota et al. [8], the mean interval from fusion to revision surgery for ASD is approximately 51 months, ranging from 41.3 to 62.4 months. We excluded studies with limited followup time less than 24 months and the occurrence of ASD is greater with longer follow-up.

4.4. Biomechanical Characteristics in Dynamic Devices on Spine Range of Motion (ROM) and Intradiscal Pressure (IDP). Schmoelz et al. [38] reported Dynesys does not change IDP at proximal adjacent disc after fixation under momentcontrolled mode, while Cabello et al. [39] reported Dynesys decreases $50 \%$ of the IDP at instrumented level and increases $10 \%$ of the IDP at supra-adjacent level under load-controlled mode. Different controlled modes in biomechanical testing may explain these diverse results [40]. Moreover, Schmoelz et al. [38] reported Dynesys is more flexible than rigid fixation, but spine ROM was still limited.

Lafage et al. [41] reported that the Wallis decreases the disc stress and ROM and increases the spinous process loading at instrumented level. Bellini et al. [31] reported that DIAM in vitro decreases ROM and IDP at instrumented level. The Wallis and DIAM both decrease but preserve some degree of ROM [31, 41], which can decrease the stresses at the adjacent level.

4.5. Rationale of "Topping-Off" Technique and Clinical Application. The "topping-off" technique provides a transitional zone between caudal rigid fused segment and cephalad mobile unfused spines, which may decrease the incidence of ASD $[10,11]$. Based on posterior dynamic stabilization system reported by Khoueir et al. [12], the Dynesys construct belongs to hybrid stabilization device; Wallis and DIAM belong to posterior interspinous device. Similar biomechanical characteristics include decreased IDP and limited [31, 38, 39, 41, 42] but still preserve some ROM at HSP/IPD instrumented level. 


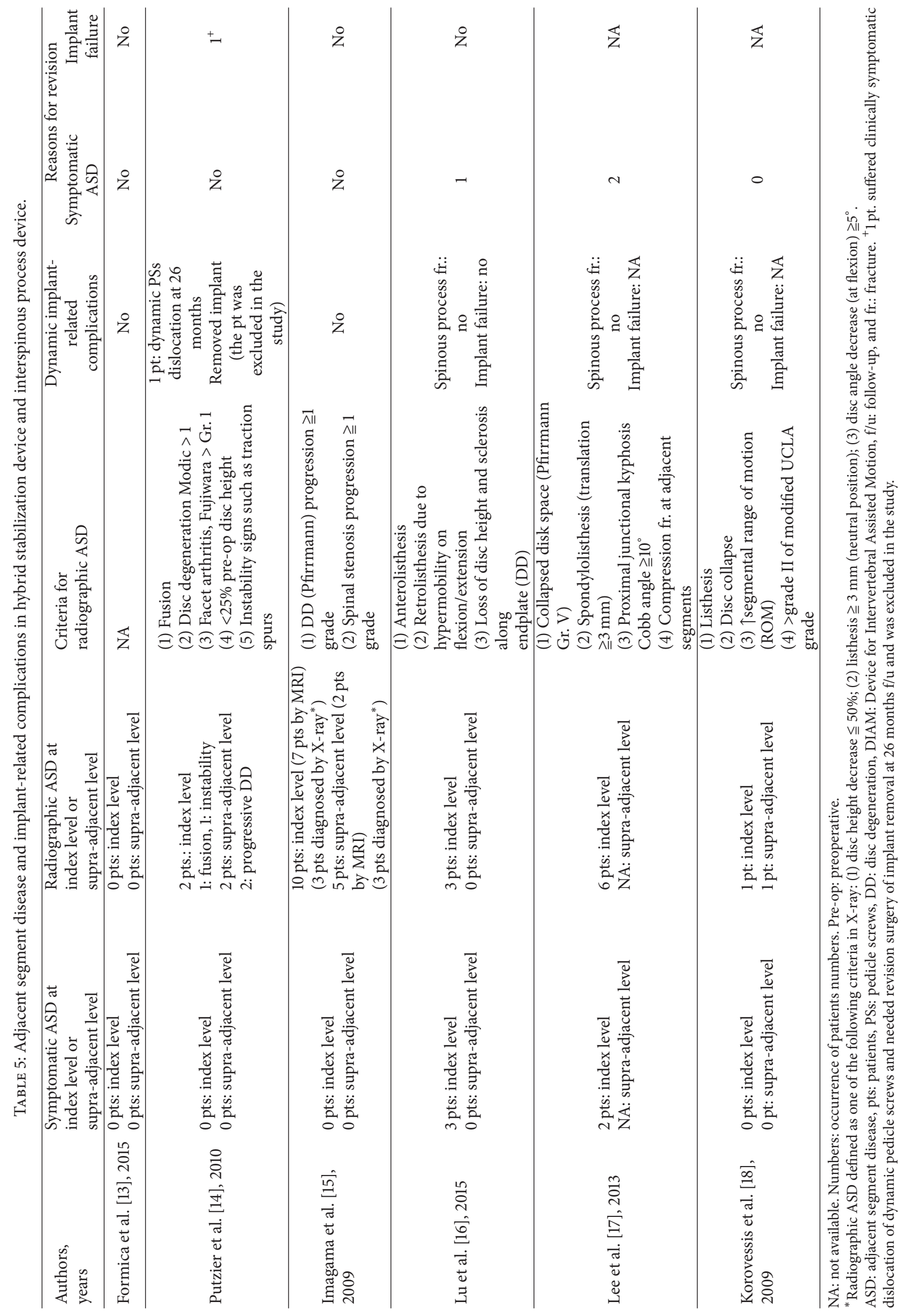




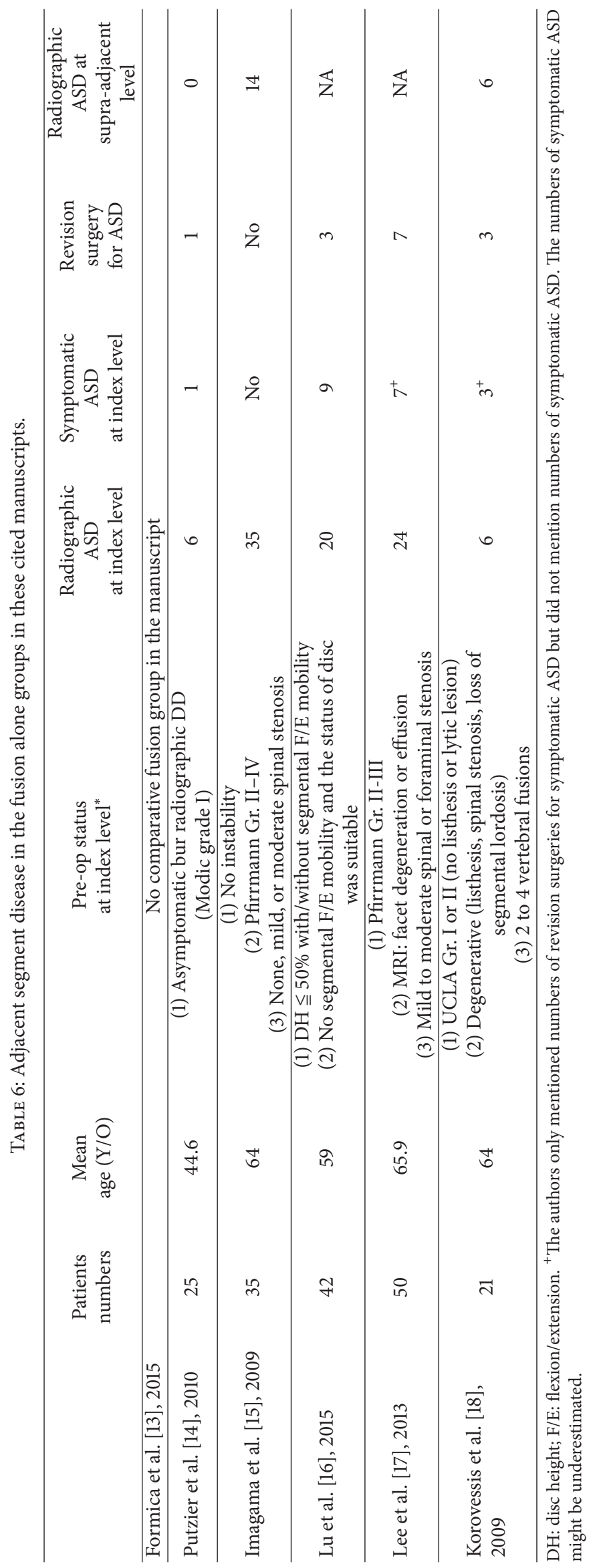


Based on this systematic review, the incidences of radiographic ASD at index level were $12.6 \%, 10.2 \%$, and $52.6 \%$ in HSD, IPD, and fusion alone, respectively. With the "toppingoff" technique, the incidence of ASD seems to decrease significantly at mid-term follow-up. These devices might possibly alleviate the degenerative progression above the fusion level. Regarding the incidence of radiographic ASD at supra-adjacent level, there were $1 \%, 10.5 \%$, and $24.7 \%$ in IPD, HSD, and fusion alone, respectively. The IPD has the best result in delayed progression of ASD at supra-adjacent level. From the biomechanical view, we assumed that the HSD was more rigid than IPD but less rigid than the instrumented fusion, which may be one of the explanations for the results. Another possible reason for higher incidence of ASD at supra-adjacent level in HSD comparing to IPD is that iatrogenic facet joints surface might jeopardize when placing proximal pedicle screws $[8,43]$. More in vitro biomechanical and high-quality prospective randomized studies are needed for further clarification on the issue.

4.6. Implants- (HSD or IPD) Related Complications. The incidence of broken pedicle screws in treatment of degenerative lumbar disease ranged from $2.2 \%$ to $12.4 \%$ [44-46] based on either total pedicle screws or patient numbers. In our results, 2 broken dislodged dynamic screws in 1 patients $(0.98 \%, 1$ out of 102 patients) in HSD group were observed, which was much lower than traditional pedicle screws fixation. This result could be different if more studies were to be analyzed or if follow-up was longer.

After Wallis being implanted, there is a change in the stress distribution of the spine, especially the spinous process [41]. Moreover, application of the tension band construct significantly increases the stress of the contact surface between the spinous process and the implant. Significant bone resorption was observed in more than $50 \%$ of the patients with Wallis implantation as reported by Wang et al. [47] and by Miller et al. [48]. The possible reasons to explain spinous process fracture or resorption are as follows: (1) the downward conduction of stress in the lumbar spine at greatest force at L5 spinous process [49]; (2) continuous motion at implanted level. Nevertheless, neither bone resorption nor spinous fracture was observed in this review.

4.7. Can Preoperative Disc Degeneration Affect the Incidence of ASD after Fusion? Park et al. [4] reported that the preoperative condition of adjacent disc for further implication in ASD following fusion is still elusive. Ghiselli et al. [3] reported the correlation between ASD and preoperative disc degeneration status at the time of surgery is not significant in 215 patients based on UCLA disc degeneration grading with mean 7-year follow-up. Nakai et al. [36] and Liang et al. [50] reported the preoperative disc degeneration correlates with the progression of ASD at adjacent fusion level based on the disc height and pre-MRI Pfirrmann's grading, respectively. All these studies did not perform the postoperative MRI image to evaluate the disc degenerative status as final follow-up.

Preoperative disc degeneration with Pfirrmann grade $\geqq$ III $[50,51]$ has a higher chance of developing symptomatic ASD. Regarding relative risks (RR) for developing ASD after fusion surgery, Ghiselli et al. [3] reported L4-5 poses a high risk, T12-L1, L1-2, and L3-4 have the intermediate risks, and L2-3 has the lower relative risks. Liang et al. [50] reported disc bulge in preoperative CT examination may serve as reasonable prediction for symptomatic ASD. Sénégas [52] reported that Wallis can be used for disc degeneration of Pfirrmann's classification grades II, III, and IV above the fusion level.

Taken together, surgeons should be more aware of preoperative adjacent disc condition. The reasonable indications for "topping-off technique" might be (1) Pfirrmann Gr. $\geqq$ III, (2) budged disc, and (3) high risk (L4-5) disc level and relative intermediate risk disc levels (T12-L1, L1-2, and L34). However, we found inconclusive surgical indications for topping-off fixation in this systematic review. We still need more evidence to support this conclusion by prospective randomized controlled study. Nevertheless, we suggest surgeons to pay more attention to the preoperative adjacent disc degenerative status, correlating between radiographic findings and patients' symptoms. The patient should be informed on the controversial nature and unpredictable outcomes when inserting these devices. More importantly, surgeons could improve their surgical techniques, such as maintaining the lordosis at the instrumented levels $[5,25,26]$, no violation of the proximal adjacent facet joints $[53,54]$ when placing pedicle screws at the most upper levels, and no excessive distraction of disc for interbody fusion [55]. These techniques will likely lessen the development of ASD.

4.8. Limitations. Several major drawbacks or limitations were found in this systematic review. First, a small number of enrolled patients and short follow-up time do not lead to a definitive conclusion. Second, there could be selection bias. Third, the criteria of radiographic parameters for ASD were not consistent in these cited studies. We suggest using MRI images combined with flexion-extension radiography to diagnose ASD if feasible. Based on our literature review, the application of "topping-off" technique with HSD or IPD above fusion to avoid ASD still lacks good evidence, and therefore prospective randomized clinical trials should be conducted to further elucidate the role of topping-off techniques.

\section{Conclusion}

Although the evidence is weak, the "topping-off" technique with HSD or IPD might decrease the incidence of proximal ASD both radiographically and symptomatically as compared to the fusion group. At the index level, the effects of HSD or IPD for decreasing ASD were similar. At supra-adjacent level, IPD seems to have the better effect of avoiding ASD. In conclusion, the "topping-off" technique might be considered as a possible solution for postfusion ASD, but further research is needed prior to wide application. The patient selection and choices of stabilizing implants should be assessed with more level I clinical studies. Based on our literatures review, the preventive strategy of ASD with application of "toppingoff" technique above fusion is still elusive, and prospective randomized trials with higher quality are still required for 
further elucidating the effect of topping-off technique for prevention of ASD.

\section{Competing Interests}

The authors declare that there is no conflict of interests regarding the publication of this paper.

\section{References}

[1] K. K. Lingutla, R. Pollock, E. Benomran et al., "Outcome of lumbar spinal fusion surgery in obese patients: a systematic review and meta-analysis," The Journal of Bone \& Joint SurgeryBritish Volume, vol. 97, no. 10, pp. 1395-1404, 2015.

[2] L. Y. Carreon, S. D. Glassman, and J. Howard, "Fusion and nonsurgical treatment for symptomatic lumbar degenerative disease: a systematic review of Oswestry Disability Index and MOS Short Form-36 outcomes," Spine Journal, vol. 8, no. 5, pp. 747-755, 2008.

[3] G. Ghiselli, J. C. Wang, N. N. Bhatia, W. K. Hsu, and E. G. Dawson, "Adjacent segment degeneration in the lumbar spine," The Journal of Bone and Joint Surgery A, vol. 86, no. 7, pp. 14971503, 2004.

[4] P. Park, H. J. Garton, V. C. Gala, J. T. Hoff, and J. E. McGillicuddy, "Adjacent segment disease after lumbar or lumbosacral fusion: review of the literature," Spine, vol. 29, no. 17, pp. 1938-1944, 2004.

[5] M. Kumar, A. Baklanov, and D. Chopin, "Correlation between sagittal plane changes and adjacent segment degeneration following lumbar spine fusion," European Spine Journal, vol. 10, no. 4, pp. 314-319, 2001.

[6] F. M. Phillips, G. D. Carlson, H. H. Bohlman, and S. S. Hughes, "Results of surgery for spinal stenosis adjacent to previous lumbar fusion," Journal of Spinal Disorders, vol. 13, no. 5, pp. 432-437, 2000.

[7] W. J. Chen, P. L. Lai, C. C. Niu, L. H. Chen, T. S. Fu, and C. B. Wong, "Surgical treatment of adjacent instability after lumbar spine fusion," Spine, vol. 26, no. 22, pp. E519-E524, 2001.

[8] Y. Aota, K. Kumano, and S. Hirabayashi, "Postfusion instability at the adjacent segments after rigid pedicle screw fixation for degenerative lumbar spinal disorders," Journal of Spinal Disorders and Techniques, vol. 8, no. 6, pp. 464-473, 1995.

[9] S. Etebar and D. W. Cahill, "Risk factors for adjacent-segment failure following lumbar fixation with rigid instrumentation for degenerative instability," Journal of Neurosurgery, vol. 90, no. 4, pp. 163-169, 1999.

[10] H.-Y. Liu, J. Zhou, B. Wang et al., "Comparison of toppingoff and posterior lumbar interbody fusion surgery in lumbar degenerative disease: a retrospective study," Chinese Medical Journal, vol. 125, no. 22, pp. 3942-3946, 2012.

[11] Z. Zhu, C. Liu, K. Wang et al., "Topping-off technique prevents aggravation of degeneration of adjacent segment fusion revealed by retrospective and finite element biomechanical analysis," Journal of Orthopaedic Surgery and Research, vol. 10, article no. 10, 2015.

[12] P. Khoueir, K. A. Kim, and M. Y. Wang, "Classification of posterior dynamic stabilization devices," Neurosurgical focus, vol. 22, no. 1, 2007.

[13] M. Formica, L. Cavagnaro, M. Basso, A. Zanirato, L. Felli, and C. Formica, "Is it possible to preserve lumbar lordosis after hybrid stabilization? Preliminary results of a novel rigid-dynamic stabilization system in degenerative lumbar pathologies," European Spine Journal, vol. 24, pp. 849-854, 2015.

[14] M. Putzier, E. Hoff, S. Tohtz, C. Gross, C. Perka, and P. Strube, "Dynamic stabilization adjacent to single-level fusion: part II. No clinical benefit for asymptomatic, initially degenerated adjacent segments after 6 years follow-up," European Spine Journal, vol. 19, no. 12, pp. 2181-2189, 2010.

[15] S. Imagama, N. Kawakami, Y. Matsubara, T. Kanemura, T. Tsuji, and T. Ohara, "Preventive effect of artificial ligamentous stabilization on the upper adjacent segment impairment following posterior lumbar interbody fusion," Spine, vol. 34, no. 25, pp. 2775-2781, 2009.

[16] K. Lu, P.-C. Liliang, H.-K. Wang et al., "Reduction in adjacentsegment degeneration after multilevel posterior lumbar interbody fusion with proximal DIAM implantation," Journal of Neurosurgery: Spine, vol. 23, no. 2, pp. 190-196, 2015.

[17] C.-H. Lee, S.-J. Hyun, K.-J. Kim, T.-A. Jahng, S. H. Yoon, and H.-J. Kim, "The efficacy of lumbar hybrid stabilization using the DIAM to delay adjacent segment degeneration: an intervention comparison study with a minimum 2 -year followup," Neurosurgery, vol. 73, no. 2, pp. ons224-ons232, 2013.

[18] P. Korovessis, T. Repantis, S. Zacharatos, and A. Zafiropoulos, "Does Wallis implant reduce adjacent segment degeneration above lumbosacral instrumented fusion?" European Spine Journal, vol. 18, no. 6, pp. 830-840, 2009.

[19] N. Panic, E. Leoncini, G. De Belvis, W. Ricciardi, and S. Boccia, "Evaluation of the endorsement of the preferred reporting items for systematic reviews and meta-analysis (PRISMA) statement on the quality of published systematic review and meta-analyses," PLOS ONE, vol. 8, Article ID e83138, 2013.

[20] L. Shamseer, D. Moher, M. Clarke et al., "Preferred reporting items for systematic review and meta-analysis protocols (PRISMA-P) 2015: elaboration and explanation," BMJ, vol. 349, Article ID g7647, 2015.

[21] "The National Heart Lung and Blood Institute Quality assessment tool for case series studies," https://www.nhlbi.nih.gov/ health-pro/guidelines/in-develop/cardiovascular-risk-reduction/tools/case_series.

[22] C. S. Lee, C. J. Hwang, S. W. Lee et al., "Risk factors for adjacent segment disease after lumbar fusion," European Spine Journal, vol. 18, article 1637, 2009.

[23] A. F. Mannion, G. Leivseth, J.-I. Brox, P. Fritzell, O. Hägg, and J. C. T. Fairbank, "ISSLS prize winner: long-term followup suggests spinal fusion is associated with increased adjacent segment disc degeneration but without influence on clinical outcome: results of a combined follow-up from 4 randomized controlled trials," Spine, vol. 39, no. 17, pp. 1373-1383, 2014.

[24] M. D. Rahm and B. B. Hall, "Adjacent-segment degeneration after lumbar fusion with instrumentation: a retrospective study," Journal of Spinal Disorders, vol. 9, no. 5, pp. 392-400, 1996.

[25] J. D. Schlegel, J. A. Smith, and R. L. Schleusener, "Lumbar motion segment pathology adjacent to thoracolumbar, lumbar, and lumbosacral fusions," Spine, vol. 21, no. 8, pp. 970-981, 1996.

[26] S. Umehara, M. R. Zindrick, A. G. Patwardhan et al., "The biomechanical effect of postoperative hypolordosis in instrumented lumbar fusion on instrumented and adjacent spinal segments," Spine, vol. 25, no. 13, pp. 1617-1624, 2000.

[27] H.-J. Wilke, P. Neef, M. Caimi, T. Hoogland, and L. E. Claes, "New in vivo measurements of pressures in the intervertebral disc in daily life," Spine, vol. 24, no. 8, pp. 755-762, 1999. 
[28] C. Wimmer, H. Gluch, M. Krismer, M. Ogon, and R. Jesenko, "AP-translation in the proximal disc adjacent to lumbar spine fusion," Acta Orthopaedica Scandinavica, vol. 68, no. 3, pp. 269272, 1997.

[29] J. Y. Yang, J. Lee, and H. Song, "The impact of adjacent segment degeneration on the clinical outcome after lumbar spinal fusion," Spine, vol. 33, no. 5, pp. 503-507, 2008.

[30] L. L. Wiltse, S. E. Radecki, H. M. Biel et al., "Comparative study of the incidence and severity of degenerative change in the transition zones after instrumented versus noninstrumented fusions of the lumbar spine," Journal of Spinal Disorders, vol. 12, no. 1, pp. 27-33, 1999.

[31] C. M. Bellini, F. Galbusera, M. T. Raimondi, G. V. Mineo, and M. Brayda-Bruno, "Biomechanics of the lumbar spine after dynamic stabilization," Journal of Spinal Disorders \& Techniques, vol. 20, no. 6, pp. 423-429, 2007.

[32] B. W. Cunningham, Y. Kotani, P. S. McNulty, A. Cappuccino, and P. C. McAfee, "The effect of spinal destabilization and instrumentation on lumbar intradiscal pressure: an in vitro biomechanical analysis," Spine, vol. 22, no. 22, pp. 2655-2663, 1997.

[33] S. L. Weinhoffer, R. D. Guyer, M. Herbert, and S. L. Griffith, "Intradiscal pressure measurements above an instrumented fusion a cadaveric study," Spine, vol. 20, no. 5, pp. 526-531, 1995.

[34] M. F. Hambly, L. L. Wiltse, N. Raghavan, G. Schneiderman, and C. Koenig, "The transition zone above a lumbosacral fusion," Spine, vol. 23, no. 16, pp. 1785-1792, 1998.

[35] M. Penta, A. Sandhu, and R. D. Fraser, "Magnetic resonance imaging assessment of disc degeneration 10 years after anterior lumbar interbody fusion," Spine, vol. 20, no. 6, pp. 743-747, 1995.

[36] S. Nakai, H. Yoshizawa, and S. Kobayashi, "Long-term followup study of posterior lumbar interbody fusion," Journal of Spinal Disorders, vol. 12, no. 4, pp. 293-299, 1999.

[37] M. N. Kumar, F. Jacquot, and H. Hall, "Long-term follow-up of functional outcomes and radiographic changes at adjacent levels following lumbar spine fusion for degenerative disc disease," European Spine Journal, vol. 10, no. 4, pp. 309-313, 2001.

[38] W. Schmoelz, J. F. Huber, T. Nydegger, Dipl-Ing, L. Claes, and H. J. Wilke, "Dynamic stabilization of the lumbar spine and its effects on adjacent segments: an in vitro experiment," Journal of Spinal Disorders and Techniques, vol. 16, no. 4, pp. 418-423, 2003.

[39] J. Cabello, J. M. Cavanilles-Walker, M. Iborra, M. T. Ubierna, A. Covaro, and J. Roca, "The protective role of dynamic stabilization on the adjacent disc to a rigid instrumented level. An in vitro biomechanical analysis," Archives of Orthopaedic and Trauma Surgery, vol. 133, no. 4, pp. 443-448, 2013.

[40] P. Strube, S. Tohtz, E. Hoff, C. Gross, C. Perka, and M. Putzier, "Dynamic stabilization adjacent to single-level fusion: part I. Biomechanical effects on lumbar spinal motion," European Spine Journal, vol. 19, no. 12, pp. 2171-2180, 2010.

[41] V. Lafage, N. Gangnet, J. Sénégas, F. Lavaste, and W. Skalli, "New interspinous implant evaluation using an in vitro biomechanical study combined with a finite-element analysis," Spine, vol. 32, no. 16, pp. 1706-1713, 2007.

[42] H.-J. Wilke, J. Drumm, K. Häussler, C. MacK, W.-I. Steudel, and A. Kettler, "Biomechanical effect of different lumbar interspinous implants on flexibility and intradiscal pressure," European Spine Journal, vol. 17, no. 8, pp. 1049-1056, 2008.

[43] B. He, L. Yan, H. Guo, T. Liu, X. Wang, and D. Hao, "The difference in superior adjacent segment pathology after lumbar posterolateral fusion by using 2 different pedicle screw insertion techniques in 9-year minimum follow-up," Spine, vol. 39, no. 14, pp. 1093-1098, 2014.

[44] N. Boos and J. K. Webb, "Pedicle screw fixation in spinal disorders: a European view," European Spine Journal, vol. 6, pp. 2-18, 1997.

[45] P. C. Jutte and R. M. Castelein, "Complications of pedicle screws in lumbar and lumbosacral fusions in 105 consecutive primary operations," European Spine Journal, vol. 11, no. 6, pp. 594-598, 2002.

[46] J. E. Lonstein, F. Denis, J. H. Perra, M. R. Pinto, M. D. Smith, and R. B. Winter, "Complications associated with pedicle screws," The Journal of Bone \& Joint Surgery - American Volume, vol. 81, no. 11, pp. 1519-1528, 1999.

[47] K. Wang, Z. Zhu, B. Wang, Y. Zhu, and H. Liu, "Bone resorption during the first year after implantation of a single-segment dynamic interspinous stabilization device and its risk factors," BMC Musculoskeletal Disorders, vol. 16, article no. 117, 2015.

[48] J. D. Miller, M. C. Miller, and M. G. Lucas, "Erosion of the spinous process: a potential cause of interspinous process spacer failure-report of 2 cases," Journal of Neurosurgery: Spine, vol. 12, no. 2, pp. 210-213, 2010.

[49] L. M. Schulte, J. R. O’Brien, L. E. Matteini, and W. D. Yu, "Change in sagittal balance with placement of an interspinous spacer," Spine, vol. 36, no. 20, pp. E1302-E1305, 2011.

[50] J. Liang, Y. Dong, and H. Zhao, "Risk factors for predicting symptomatic adjacent segment degeneration requiring surgery in patients after posterior lumbar fusion," Journal of Orthopaedic Surgery and Research, vol. 9, article 97, 2014.

[51] H. Ishihara, M. Kanamori, Y. Kawaguchi, H. Nakamura, and T. Kimura, "Adjacent segment disease after anterior cervical interbody fusion," The Spine Journal, vol. 4, no. 6, pp. 624-628, 2004.

[52] J. Sénégas, "Mechanical supplementation by non-rigid fixation in degenerative intervertebral lumbar segments: the wallis system," European Spine Journal, vol. 11, supplement 2, pp. S164S169, 2002.

[53] S. M. Jones-Quaidoo, M. Djurasovic, R. K. Owens III, and L. Y. Carreon, "Superior articulating facet violation: percutaneous versus open techniques," Journal of Neurosurgery: Spine, vol. 18, no. 6, pp. 593-597, 2013.

[54] D. Lau, S. W. Terman, R. Patel, F. L. Marca, and P. Park, "Incidence of and risk factors for superior facet violation in minimally invasive versus open pedicle screw placement during transforaminal lumbar interbody fusion: a comparative analysis: clinical article," Journal of Neurosurgery: Spine, vol. 18, no. 4, pp. 356-361, 2013.

[55] T. Kaito, N. Hosono, Y. Mukai, T. Makino, T. Fuji, and K. Yonenobu, "Induction of early degeneration of the adjacent segment after posterior lumbar interbody fusion by excessive distraction of lumbar disc space: clinical article," Journal of Neurosurgery: Spine, vol. 12, no. 6, pp. 671-679, 2010. 


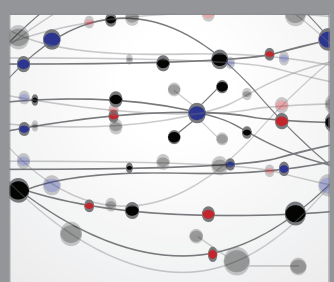

The Scientific World Journal
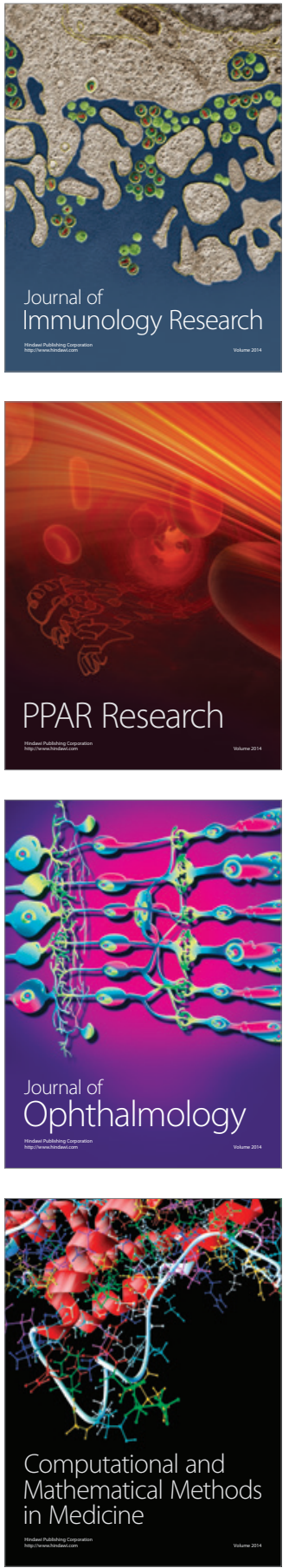

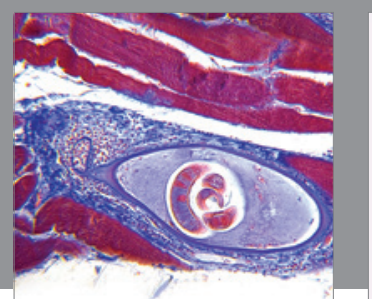

Gastroenterology Research and Practice
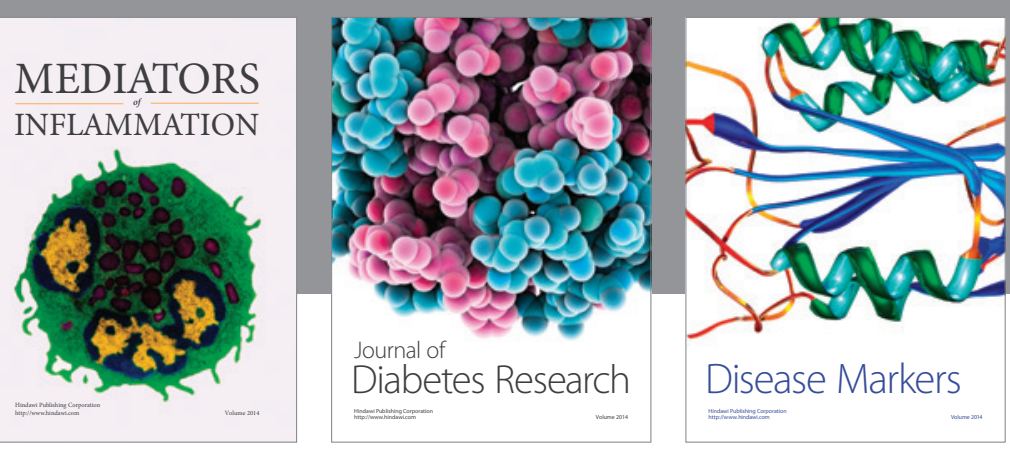

Disease Markers

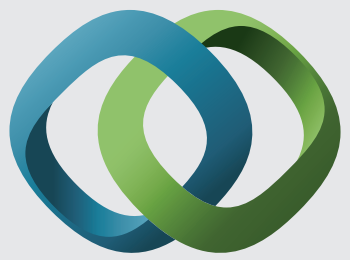

\section{Hindawi}

Submit your manuscripts at

https://www.hindawi.com
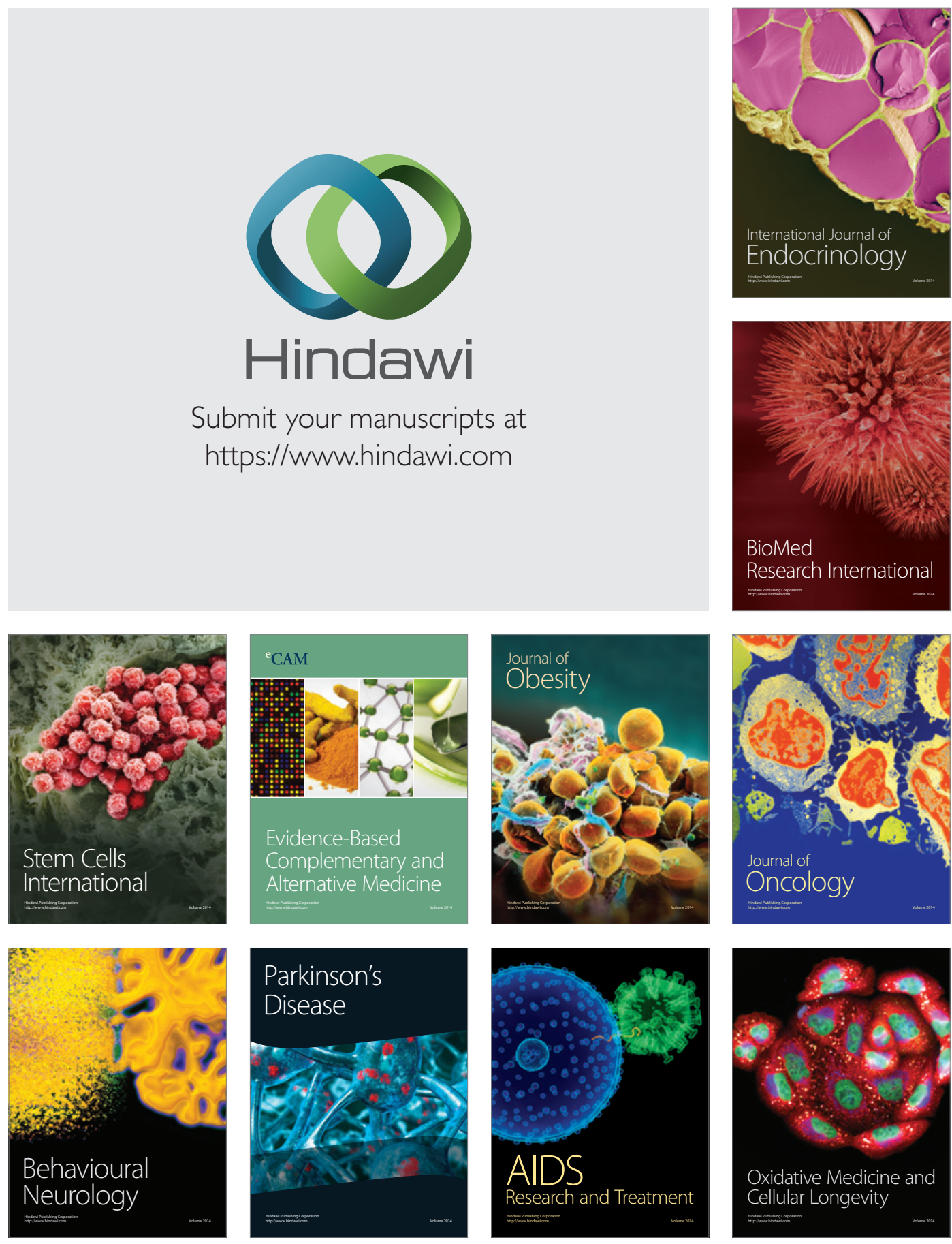